\title{
Telling one particle from another
}

\author{
An argument about the proper derivation of the Maxwell-Boltzmann distribution raises questions of \\ the distinguishability of particles with a bearing on whether quantum mechanics is really classical.
}

What the textbooks say about the distribution of particles (which may be atoms) among the various energy states accessible to them may be over-simple. That is the most obvious conclusion to be drawn from an article by Domenico Costantini of the University of Genoa in Physics Letters A for 7 September (123, 433; 1987). The article is a much-delayed riposte to that of $\mathrm{J}$. Tersoff of Bell Laboratories (before it was renamed AT\&T Bell Laboratories) and David Bayer of Columbia University (Phys. Rev. Lett. 50, 553; 1983).

The underlying issue is whether, and in what circumstances, particles can be regarded as distinguishable from each other. The general opinion, of course, is that classical particles are distinguishable from each other, but that quantum particles are not. There are still many zealous men and women who hope to show that quantum particles are merely classical particles in disguise.

The best place to start is with the textbooks, and with a system consisting of $N$ particles each of which may be in any one of $M$ different states. A particular (microscopic) state of the system may be represented by specifying the state in which each particle is to be found; another is to say how many particles there are in each of the $M$ states.

Plainly the second description is more general than the first. The second may, for example, be specified by a set of numbers $n$ which count the numbers of particles in the state whose index is $i$ (running from 1 to $M$ ). Obviously this set of occupation numbers must always satisfy the condition that their total is $N$. The system must also satisfy the condition that its total energy is fixed.

The crucial step in the Boltzmann argument is to calculate the mean state of the system (and also its fluctuations), which entails the calculation of the probability distribution of the statistic $n_{i}$. Luckily, as Boltzmann discovered, there are some simplifying assumptions that can plausibly be made. If (as they were in his time) all particles are distinguishable, but if there can be no physical difference between states of the system in which individual particles are interchanged, it is possible to set about the task of calculating the likelihood of particular macroscopic states (specified by sets of occupation numbers $n_{i}$ ).
The problem reduces to that of calculating the number of ways in which a group of $N$ marbles (or objects of any other kind) may be distributed among $M$ containers in such a way that there are $n$, in the first, $n$, in the second, and so on. The standard high-school answer is that the number of ways in which this may be done is $N ! / n, ! n, \ldots$ where the denominator of this fraction is the product of all the factorial functions of the occupancy numbers. Such a configuration is known as a "complexion" in the language of statistical mechanics.

The usual treatment of the problem is to find the state with the greatest likelihood subject to the constraint that the number of particles is given and that the energy is fixed. It is an essential part of the arithmetic that the particles are distinguishable from each other, for otherwise the numbers would not turn out to be the coefficients of the multinomial expansion (the obvious generalization of the binomial expansion) that leads, for example, to Boltzmann's conclusion that the mean value of the occupancy number is a decreasing exponential function of the inverse temperature.

If it is assumed that particles are indistinguishable from each other, the combinatorial coefficients come out differently and Bose-Einstein statistics are the consequence. The further assumption that two particles cannot be in the same state (after allowing for the possibility of spin) leads to Fermi-Dirac statistics.

That is what we all know. The surprise is that Costantini shows that we may be wrong. He demonstrates that, while the textbook argument and its assumptions may be sufficient, they are by no means necessary. Indeed, he argues, it is also sufficient (in the sense in which mathematicians use the word) to assume that the probability that a particular particle will be found in a particular state is independent of the states in which the other particles have already been assigned to states (subject only to the energy constraint) and that the identity of the particle itself is irrelevant.

Going further, Costantini sets out to replace even these sparse assumptions by what is, in effect, a set of axioms such as Euclid might have devised. Stripped of its subtleties (of which there many), the argument boils down to the assertion that the probabilities of the occurrence of particular configurations are algebraically symmetric with respect to the interchange of particles - which is not the same thing as saying that the particles are distinguishable.

But if it is not necessary to suppose that particles are distinguishable to arrive at Maxwell-Boltzmann statistics, from what do quantum statistics (Bose-Einstein or Fermi-Dirac) arise? In operational terms, the different possibilities correspond to different ways of choosing the numerical constant in optimization problems subject to a constraint, as in almost any variational problem.

Two features of this argument need comment, of which the most obvious is the contradiction between Costantini and Tersoff \& Bayer. Taking a similarly ascetic point of view, the latter set out to show that the assumption in quantum statistical mechanics that particles are indistinguishable can be traded for the assumption that, even with the energy constraint, different configurations (represented by different sets of numbers $n_{i}$ ) will not be equally likely to arise. They say the assumption is plausible, but it is not self-evidently simple.

Two important questions arise, of which the most obvious is that even to those (like most of us) who side with Costantini in believing that quantum mechanics and classical mechanics are different, there must surely be ways in which the question of whether particles are distinguishable or not should be settled by an appeal to the quantum theory of measurement. After sixty years of the Heisenberg Uncertainty Principle, one would expect there to be a way of telling what recipe should be used in what circumstances.

There is also a question of inference to be settled. The Maxwell-Boltzmann distribution is evidently a good representation of reality in the sense that it works. But how rigorously does it have to be tested before it can be taken to be a kind of truth? On the face of things, Costantini's paper is strong support for the proposition that the distribution is reality, but then there arises the nagging doubt that a description of the real world derived from mere axioms may be a special case of something more general and more simple.

John Maddox 